\title{
Does the Middle Turbinate Share the Clinical and Pathological Changes with Paranasal Sinuses in Chronic Rhinosinusitis?
}

Hamza Elshafie Ahmed ${ }^{1}$, Mahmoud Farouk Sherif ${ }^{2}$, Zeinab Salah El-Deen Mohammed ${ }^{1}$, Mostafa Osman Ramadan ${ }^{1}$

1. ENT Department. Faculty of medicine/Asyut University/Asyut/Egypt.

2. Pathology Department. Faculty of medicine/Asyut University/Asyut/Egypt.

\begin{abstract}
Introduction: Chronic rhinosinusitis with nasal polyposis (CRSwNP) is an inflammation of the nose and paranasal sinus (PNS) mucosa for at least 12 weeks with the formation of nasal polyps. This is a multi-factorial disease. Nasal polyposis represents $4 \%$ of the population. Few studies have looked at the histopathological changes in ethmoid mucosa and compared them with those of the mucosa of the nasal septum, inferior turbinate (IT), and middle turbinate (MT) in patients with CRS.
\end{abstract}

Objective: To compare clinical and histopathological findings of the middle turbinate and ethmoidal polyp in CRSwNP patients refractory to medical treatment.

Methods: Forty patients with CRSwNP refractory to treatment were included. Endoscopic and histopathological findings of MT and ethmoid sinus samples were compared.

Results: By endoscopic(macroscopic) examination, there was a statistically significant difference between MT and ethmoidal polyp. Polyps in the ethmoid were present in all cases. Polypoidal MT mucosa was present in a quarter of patients. In the rest of the patients, the MT mucosa was edematous or normal. By microscopic examination, there was no statistically significant difference between the two sites in the degree of basement membrane (BM) thickness, goblet cell hyperplasia, eosinophils, and polymorphs. Statistically, a significant difference was present in the degree of sub-epithelial edema, the number of sub-mucous glands, and lymphocytes.

Conclusion: Although MT does not share the macroscopic changes with the ethmoid sinus in patients with CRSwNP, at the microscopic level, both sites share the pathologic changes to a far extent. It is better to address the MT in the medical and surgical management of CRSwNP for better results.

Keywords: Chronic rhinosinusitis with nasal polyposis, Nasal polyposis, middle turbinate

\section{Introduction:}

Chronic rhinosinusitis (CRS) with nasal polyposis is defined as inflammation of the nose and paranasal sinus mucosa for at least 12 weeks with polyp formation. It is considered a multifactorial disease, with a possible role of mucociliary dysfunction, infection, allergy, and aspirin intolerance. The underlying mechanism interlinking these pathologic conditions to polyp formation is still unclear ${ }^{1}$.
The term rhinosinusitis has been proposed instead of sinusitis to refer to inflammation of the paranasal sinuses (PNS) by the American Academy of Otolaryngology-Head and Neck Surgery Foundation in $1997^{2}$.

Chronic rhinosinusitis negatively affects the patient's quality of life in different ways. There is also a potential for some complications. Treatment of CRSwNP may be achieved by drug administration. Failure of pharmacotherapy is an indication of 
surgical management. There is always a possibility of disease recurrence after surgery ${ }^{3}$. Recurrence of sinonasal polyposis after endoscopic sinus surgery (ESS) is relatively high. It is estimated to be around $40 \%$ after 18 months ${ }^{4}$.

Few studies have compared the inflammatory changes of the nasal mucosa and the mucosa of PNS in patients with CRSwNP through microscopic examination of the ethmoid mucosa and the mucosa of the nasal septum, IT, and MT during FESS. Histopathological evidence of rhinitis associated with chronic sinusitis was confirmed in these studies $^{5-8}$.

The aim of this study was to evaluate the clinical and histopathological changes of the middle turbinate mucosa and to compare these changes with those of the ethmoidal mucosa in patients with CRSwNP.

\section{Materials and methods}

The study protocol was reviewed and approved by the medical ethics committee of Assiut College of medicine.

This prospective case series study of 40 consecutive adult patients who underwent ESS for CRSwNP refractory to medical treatment. Patients $<18$ years, patients with mucociliary disorders, and patients with the previous ESS associated with bilateral MT resection were excluded.

For the sake of better validation of the comparison, we chose to take the two biopsy samples from the same side of the nose because some studies have pointed to some role for the local anatomic factors in the development of nasal polyposis ${ }^{9}$. We preferred to take the samples from only one side of the nose to avoid undue duplication of the number of samples, considering that the inflammatory reaction in one individual would be the same on both sides.

Diagnosis of CRSwNP was done through medical history and clinical examination, including nasoenoscopy using a 0-degree endoscope under local anesthesia. Multi-slice computed tomography (CT) scan of the nose and PNS, bone and soft tissue windows, axial and coronal cuts, $2 \mathrm{~mm}$ thickness was done to confirm the diagnosis and to exclude other possibilities and comorbidities. Before scanning the nose and before submission to surgery, patients were given oral prednisolone $(1 \mathrm{mg} / \mathrm{Kg} /$ day with a maximum of 60 $\mathrm{mg}$ ) in two divided doses for two weeks ${ }^{10}$. When there was an active infection in the nose and sinuses (mucopurulent discharge in the nasal floor or middle meatus), oral amoxicillin/clavulanic acid $1 \mathrm{gm}$ was given twice daily for one week. Oral cefdinir $300 \mathrm{mg}$ twice daily was used for five days in cases of penicillin allergy.

All surgeries were done under general inhalational anesthesia delivered through endotracheal intubation in the supine position with the head slightly elevated.

The initial step was an endoscopic assessment of the MT and ethmoids for edema and polypoidal changes. Utilizing nasal scissors or throughcutting instruments, the MT sample was then taken from the polypoidal part of the MT if there was any. When the MT was not polypoidal, the sample was taken from the anteroinferior part of the MT, sparing at least $0.5 \mathrm{~cm}$ of the superior, sagittally-oriented portion as a landmark for possible future surgeries. Ethmoidectomy was done at this point using steps described by Antunes. M.B \& Kennedy. D.W ${ }^{11}$.The 
ethmoid sample was taken from the anterior or posterior ethmoid polyps.

The samples were tagged as sample (1) for the ethmoidal polyp sample and (2) for the MT samples. Samples were preserved in formalin before transmission to the pathology laboratory. In the pathology, laboratory samples were immediately fixed in $10 \%$ formalin fixative. After overnight fixation, the tissue samples were processed by automatic tissue processing until paraffin wax was embedded. The slides were prepared by cutting 4-um-thick sections and staining with hematoxylin and eosin (H\&E).

The light microscopic findings of seven histopathological points were compared between the ethmoidal polyp mucosa and middle turbinate mucosa. These points were:

1. The thickness of the basement membrane (BM).

2. Goblet cell hyperplasia.

3. Degree of sub-epithelial edema.

4. The number of the sub-mucous gland.

5. Eosinophil infiltration.

6. Lymphocyte infiltration.

7. Polymorphonuclear leukocyte (PMN) infiltration.

All histopathological examinations were performed by the second author. Histopathological evaluation was made at high power magnification $\times 100$ for BM thickening, degree of subepithelial edema, and the number of sub-mucous glands. For inflammatory cell count and goblet cell number, evaluation was made at higher power magnification $(\times 400)$. We followed Dhong et at 12 scoring system to score the grading of the seven points mentioned. In this system, eosinophil and lymphocyte infiltration were graded into five grades from 0-4, while in the remaining items, grading was four grades from 0-3.

The postoperative care for our patients was as routinely done in all ES surgeries.

\section{Statistics:}

Data obtained were documented using Microsoft word and excel 2010. Data were analyzed using (mean \pm standard deviation) for describing the age of the patients. Continuous variables were normally distributed and compared using Wilcoxon (MannWhitney) test.

All statistical calculations were performed using SPSS version 22.0(SPSS Inc, Chicago, IL). P-values less than 0.05 were considered statistically significant.

\section{Results}

A total of 40 patients with CRSwNP were included with a mean age of $34.13 \pm 10.69$ and a range of (19-70 years). Males were slightly more than females (23 Vs. 17).

Comparison of endoscopic and microscopic findings between MT and ethmoidal polyp

There was a statistically significant difference $(\mathrm{p}<0.05)$ between MT and ethmoid mucosa regarding the presence of polyp (table1). The ethmoid mucosa was polypoidal in all patients. The MT was polypoidal only in a quarter of patients. However, the MT was edematous without polyp formation in half of the patients and normal in the remaining quarter.

There was a statistical significance difference $(p<0.05)$ between MT and ethmoidal polyp in sub-epithelial edema, sub-mucous glands, lymphocyte infiltration. The degree of submucosal edema and lymphocyte infiltration tended to be more in ethmoid tissue samples than in MT 
samples. The number of the submucous gland was higher in the MT samples than in the ethmoid samples. On the other hand, there was no statistically significant difference ( $>>0.05)$ between MT mucosa and ethmoidal polyp in thickness of BM, goblet cell hyperplasia, eosinophils, and polymorphs infiltration. The median of these items was the same in both samples, as shown in table (1) and figures $(1,2,3 \& 4)$.

Table (1) shows the endoscopic and microscopic findings of the MT and ethmoidal mucosa.

\begin{tabular}{|c|c|c|c|c|c|}
\hline \multirow{2}{*}{$\begin{array}{l}\text { Points of } \\
\text { comparison } \\
\text { Endoscopic view: }\end{array}$} & \multicolumn{2}{|c|}{ Ethmoidal polyp $\mathrm{N}=40$} & \multicolumn{2}{|c|}{ MT N=40 } & \multirow{5}{*}{$\begin{array}{l}\text { P-value } \\
0.000\end{array}$} \\
\hline & $\mathrm{N}$ & $\%$ & $\mathrm{~N}$ & $\%$ & \\
\hline Polypoidal & 40 & 100.00 & 10 & 25.00 & \\
\hline $\begin{array}{l}\text { Edema } \\
\text { polyp }\end{array}$ & 0 & 0.00 & 20 & 50.00 & \\
\hline Normal & 0 & 0.00 & 10 & 25.00 & \\
\hline Microscopic view: & Median & Range & Median & Range & \\
\hline Thickness of BM & 1 & $(0-3)$ & 1 & $(0-3)$ & 0.795 \\
\hline $\begin{array}{l}\text { Goblet } \\
\text { hyperplasia }\end{array}$ & 0 & $(0-2)$ & 0 & $(0-3)$ & 0.544 \\
\hline Sub-epithelial edema & 3 & $(1-3)$ & 1 & $(0-3)$ & 0.000 \\
\hline Sub-mucous glands & 0 & $(0-2)$ & 2 & $(0-3)$ & 0.000 \\
\hline $\begin{array}{l}\text { Eosinophils } \\
\text { infiltration }\end{array}$ & 2 & $(0-4)$ & 2 & $(0-4)$ & 0.064 \\
\hline $\begin{array}{l}\text { Lymphocytes } \\
\text { infiltration }\end{array}$ & 2 & $(0-4)$ & 1 & $(0-4)$ & 0.012 \\
\hline $\begin{array}{l}\text { Polymorphs } \\
\text { infiltration }\end{array}$ & 1 & $(0-3)$ & 1 & $(0-3)$ & 0.289 \\
\hline
\end{tabular}

MT=middle turbinate. $\quad \mathrm{N}=$ number. $\quad \mathrm{BM}=$ basement membrane.

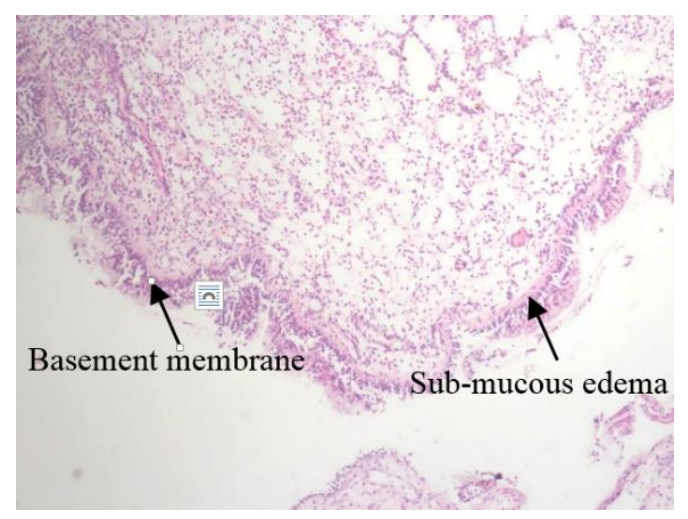

Figure 1: Section from ethmoidal polyp of a patient showing mild thickness of basement membrane (score 1) and moderate edema(score 2), (100x, H\&E).

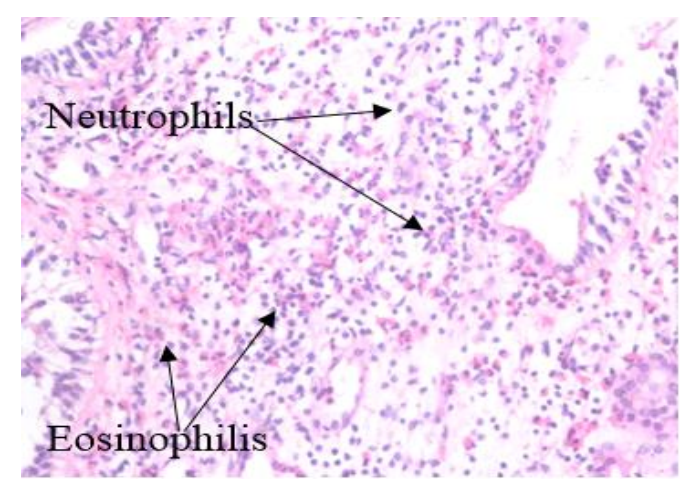

Figure 2: Section from the mucosa of MT of a patient showing heavy inflammatory (score 4), lymphocytes (score 2), and neutrophils (score1) (400x, H\&E). 


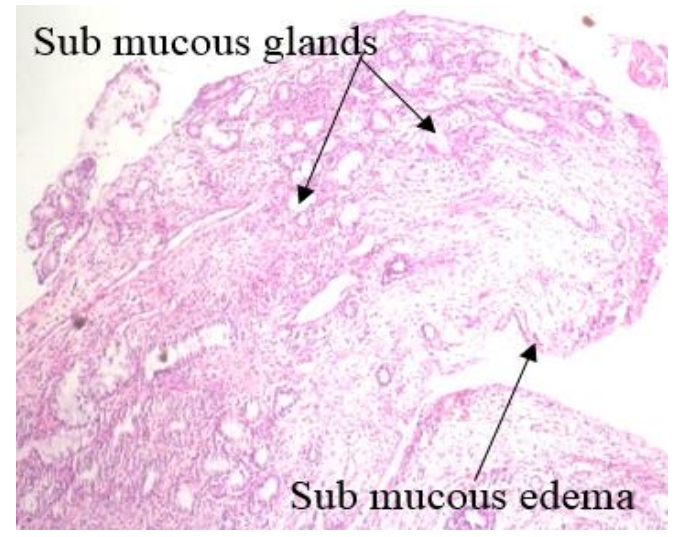

Figure 3: Section from the mucosa of MT of a patient showing mild edema (score 1) and submucosal gland hyperplasia (score 2$),\left(100 x_{2}\right.$ H\&E).

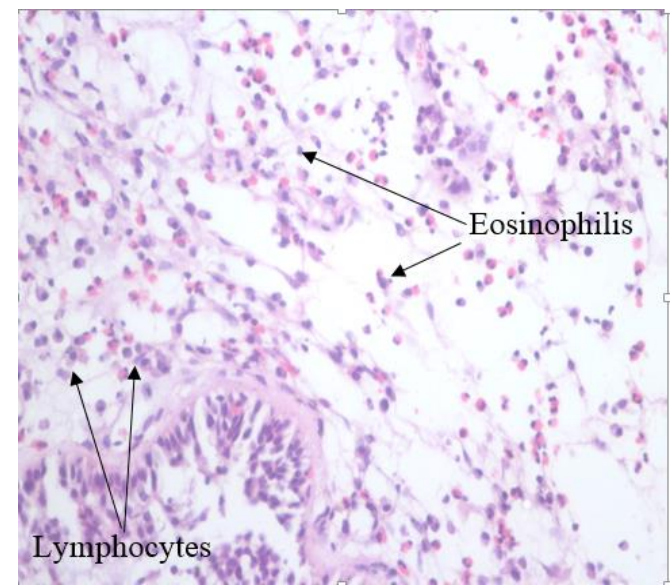

Figure 4: Section from ethmoidal polyp of a patient showing moderate inflammatory reaction with eosinophils (score 3) and mild lymphocytes infiltrate (score 1), (400x, H\&E).

\section{Discussion:}

The term chronic sinusitis has evolved and still is evolving. The early belief was that the disease is caused by infection due to obstruction of the sinus ostium. Extensive researches in this field, however, led the American Academy of Otolaryngology-Head and Neck Surgery Foundation in 1997 to propose the use of rhinosinusitis as the appropriate term for the condition ${ }^{(2)}$. In 2019, Jankowski and his group ${ }^{13}$ introduced the concept of chronic nasal dysfunction to describe various pathologic conditions that affect the nose and PNS. They divided the nose into three functional parts; olfactory, respiratory, and sinusal parts, each of which can be affected by specific diseases. Their concept did not gain popularity so far.

In this work, we were complying with the American Academy of otolaryngology head and neck surgery terminology so, we used the term chronic rhinosinusitis. Because our patients were all having nasal polyposis, we meant chronic rhinosinusitis with nasal polyps (CRSwNP) when we mentioned chronic rhinosinusitis.

We compared the mucosa of MT and ethmoids in patients with CRSwNP refractory to medical treatment at both the endoscopic and the microscopic levels.

At the endoscopic level, there was a statistically significant difference between the mucosa of both sites regarding polyp formation. The mucosa of the ethmoids was polypoidal in a significantly higher number of patients than that of the MT. Although the MT mucosa was not polypoidal in $3 / 4$ of patients, it was edematous without polyp formation in half of the patients. Considering the polypoidal change an advanced stage of edema as believed by some researchers ${ }^{14}$, hence both sites might correlate well regarding the presence of inflammatory edema, and the difference was just in the degree of the present edema (Table 1).

At the microscopic level, using H\&E-stained slides, there was no statistically significant difference between the two tissue samples in terms of the degree of BM thickening, goblet cell hyperplasia, eosinophils, and polymorphs infiltration. Such a similarity between the ethmoid and MT samples supports the concept of one continuous mucosa with a strong 
relationship between the nose and paranasal sinuses. Hence the term rhinosinusitis looks more appropriate for describing PNS diseases than sinusitis.

Statistically, a significant difference was noted, however, between the two tissue samples in the degree of submucosal edema, number of the submucosal gland, and lymphocytes infiltration.

For one reason or another, edema tended to be more marked in the ethmoids than the rest of the turbinate mucosa. We do believe -like others $\mathrm{do}^{14}$ that tissue edema is the responsible factor for the polypoidal shape of the polyps. We also believe that the marked degree of edema in the ethmoid sample was responsible for the fewer number of glands in the submucosa of the ethmoid sample. Considering a fixed number of glands in a maco-sample, the edema fluid will increase the volume of this sample and dilute that number of glands.

In one of the earliest studies comparing cellular infiltration in ethmoid and septal mucosa patients with CRS, Bhattacharyya found no significant difference in the mean noneosinophilic inflammatory cell count per high power field (HPF) between the samples from the two sites. Ethmoid mucosa -however- had a significantly higher mean eosinophil count than septal mucosa 5 .

In another study, Busaba et al. 1 compared the histopathological findings of samples taken from the ethmoid tissue and MT in patients with CRS without polyposis with the same methodology. Twenty-two consecutive patients were enrolled in that study. The total number of cellular infiltration between the two sites was very much comparable. A higher number of eosinophils and a lower number of lymphocytes were demonstrated in the ethmoid tissues. Epithelial thickness was more prominent in the ethmoid sinus samples ${ }^{8}$. Similar results were reported by Hao et al ${ }^{15}$.

Ardehali et al. in $\mathbf{2 0 1 1}^{6}$ compared the histopathological findings of samples from the ethmoid and inferior turbinate. They included 50 patients with CRSwNP. They divided their patients into two groups, those with asthma and those without asthma. The points of comparison were exactly like our points. They concluded a strong positive correlation between both tissue samples in terms of all cellular infiltration and basement membrane thickness, goblet cell hyperplasia, and sub-epithelial edema and gland formation. In our study, the cellular infiltration was comparable between the two sample sites, and the difference was significant in the sub-mucous glands number and sub-epithelial edema.

Although few, the previous studies, in general, support the concept that in patients with CRS, different parts of the nose share the ethmoid mucosa the overall inflammatory changes. The variability in the degree of this sharing could be explained by the multifactorial nature of the problem, the different anatomic sites of the nasal mucosa used for comparison, the relatively few numbers of patients used in each individual study, and the type of CRS in terms of the presence or absence of polyps.

Our results, together with those of other similar studies, stand before using the term chronic rhinosinusitis (CRS) instead of splitting it to be sinusitis and rhinitis. Our analysis also pushes to address both MT and sinus mucosa, especially during surgical management of CRS by FESS. There has been a long debate about the value 
of partly resecting the middle turbinate -especially if it looks diseased. A study in our department a few years ago showed no harm when the head of the MT was resected for the sake of accessibility, better follow-up, or to avoid lateralization with sinus outflow obstruction ${ }^{16}$. Most studies in the English literature concluded no harm with partial MT resection, with some studies even showing some benefits ${ }^{17-}$ 20.

\section{References:}

1.Slovick, A. ,Long, J., \& Hopkins ,C. (2014). Updates in the management of chronic rhinosinusitis. Clinical Practice, 11(6), 649..

2.Lanza, D. C., \& Kennedy, D. W. (1997). Adult rhinosinusitis defined. Otolaryngology-Head and Neck Surgery, 117(3_suppl), S1-S7..

3.Esmatinia , F., \& bakhshaei , M.(2014) Recurrent sinonasal polyposis after the endoscopic sinus surgery. Reviews in Clinical Medicine. ;1(2):86-92.

4.DeConde, A. S., Mace, J. C., Levy, J. M., et al (2017). Prevalence of polyp recurrence after endoscopic sinus surgery for chronic rhinosinusitis with nasal polyposis. The Laryngoscope, 127(3), 550-555..

5.Bhattacharyya, N. (2001). Chronic rhinosinusitis: is the nose really involved?. American journal of rhinology, 15(3), 169-174..

6.Ardehali, M. M., Amali, A., Bakhshaee, M., et al (2011). Histopathologic characteristics of inferior turbinate vs ethmoidal polypin chronic rhinosinusitis. Annals of diagnostic pathology, 15(4), 233-236.

7.Amali, A., Bidar, Z., Rahavi-Ezabadi, S., et al (2018). Polypoid change of middle turbinate is associated to an increased risk of polyp recurrence after surgery in patients with chronic rhinosinusitis with nasal polyps. European Archives of OtoRhino-Laryngology, 275(8), 2021-2025. .
8.Busaba, N. Y., Shin, H. J., \& Faquin, W. C. (2006). Correlation between middle turbinate and ipsilateral ethmoid histopathology in chronic rhinosinusitis. Otolaryngology - Head and Neck Surgery, 134(2), 250-254..

9.Akpinar, M., Mahmutoglu, A. S., Uçak, I., et al (2016). Anatomic variations in paranasal sinuses of patients with sinonasal polyposis: radiological evaluation. Journal of Craniofacial Surgery, 27(5), 1336-1339..

10.Martinez- Devesa, P., \& Patiar, S. (2016). Oral steroids for nasal polyps. Cochrane Database of Systematic Reviews, (7)..

11.Antunes ,M.B,. \& Kennedy ,D.W,.(2014) Surgical Management of Rhinosinusitis with and Without Polyps in Adults .Diseases of the sinuses: a comprehensive textbook of diagnosis and treatment:Chapter 23 p438-443.

12.Dhong, H. J., Kim, H. Y., \& Cho, D. Y. (2005). Histopathologic characteristics of chronic sinusitis with bronchial asthma. Acta oto-laryngologica, 125(2), 169-176.. 13.Jankowski, R., Gallet, P., Nguyen, D. T., et al (2019). Chronic rhinosinusitis of adults: new definition, new diagnosis. La Revue du praticien, 69(3), 274-278..

14.London Jr, Nyall, R., \& Reh, D. D. (2016). Differential diagnosis of chronic rhinosinusitis with nasal polyps. Rhinosinusitis with Nasal Polyposis, 79, $1-12$..

15.Hao, J., Pang, Y. T., \& Wang, D. Y. (2006). Diffuse mucosal inflammation in nasal polyps and adjacent middle turbinate. Otolaryngology-Head and Neck Surgery, 134(2), 267-275..

16.Ahmed, H. E. , \& Osman, M. M. (2016). Impact of resection of the head of the middle turbinate in endoscopic sinus surgery. Pan Arab Journal of Rhinology, 6(1), 11 ..

17.Roy, M., \& Lade, H. (2019). Functional Endoscopic Sinus Surgery of Nasal Polyposis: The Vexing Question of Whether to Resect or Preserve Middle Turbinate. Indian Journal of Otolaryngology and Head \& Neck Surgery, 71(3), 2025-2030.. 
18.Hudon, M. A., Wright, E. D., FortinPellerin, E.,et al (2018). Resection versus preservation of the middle turbinate in surgery for chronic rhinosinusitis with nasal polyposis: a randomized controlled trial. Journal of Otolaryngology-Head \& Neck Surgery, 47(1), 1-5..

19.Tan, N. C. W., Goggin, R., Psaltis, A. J., et al (2018, August). Partial resection of the middle turbinate during endoscopic sinus surgery for chronic rhinosinusitis does not lead to an increased risk of empty nose syndrome: a cohort study of a tertiary practice. In International forum of allergy \& rhinology (Vol. 8, No. 8, pp. 959-963).. 20.Halderman, A. A., Stokken, J., \& Sindwani, R. (2016, October). The effect of middle turbinate resection on topical drug distribution into the paranasal sinuses. In International forum of allergy \& rhinology (Vol. 6, No. 10, pp. 10561061).. 